Bubbles and crashes in a Black-Scholes model with delay

Appleby, J. A. D. and Riedle, M. and Swords, C.

2010

MIMS EPrint: 2010.39

Manchester Institute for Mathematical Sciences

School of Mathematics

The University of Manchester

\footnotetext{
Reports available from: http://eprints.maths.manchester.ac.uk/

And by contacting: The MIMS Secretary

School of Mathematics

The University of Manchester

Manchester, M13 9PL, UK
} 


\title{
Bubbles and crashes in a Black-Scholes model with delay
}

\author{
John A. D. Appleby* \\ School of Mathematical Sciences \\ Dublin City University \\ Glasnevin, Dublin 9, Ireland
}

\author{
Markus Riedle ${ }^{\dagger}$ \\ School of Mathematics \\ The University of Manchester \\ Oxford Road \\ Manchester M13 9PL \\ United Kingdom
}

\author{
Catherine Swords ${ }^{\ddagger}$ \\ School of Mathematical Sciences \\ Dublin City University \\ Glasnevin, Dublin 9, Ireland ${ }^{\S}$
}

May 24, 2010

\begin{abstract}
This paper studies the asymptotic behaviour of an affine stochastic functional differential equation modelling the evolution of the cumulative return of a risky security. In the model, the traders of the security determine their investment strategy by comparing short- and long-run moving averages of the security's returns. We show that the cumulative returns either obey the Law of the Iterated Logarithm, but have dependent increments, or exhibit asymptotic behaviour that can be interpreted as a runaway bubble or crash.
\end{abstract}

Mathematics Subject Classification (2000): 91B26, 91B70, 34K50, 34K25

\section{JEL Classification: G14}

Keywords: stochastic functional differential equation, resolvent, renewal equation, Brownian motion, Law of the Iterated Logarithm, Efficient Market Hypothesis

\section{Introduction}

In recent years, much attention in financial economics has focussed on the trading strategies of investors. Classical models of financial markets assume that agents are rational,

\footnotetext{
*john.appleby@dcu.ie

†markus.riedle@manchester.ac.uk

${ }^{\ddagger}$ catherine.swords2@mail.dcu.ie

$\S$ John Appleby and Markus Riedle gratefully acknowledge Science Foundation Ireland for the support of this research under the Mathematics Initiative 2007 grant 07/MI/008 "Edgeworth Centre for Financial Mathematics".
} 
have homogeneous preferences, and do not use historical market data in making their investment decisions. An important and seminal collection of papers summarising this position is Cootner [8].

Econometric evidence of market returns (e.g. Lo and MacKinlay [17]) and analysis of the behaviour of real traders reveal a more complex picture. Traders often employ rules of thumb which do not conform to notions of rational behaviour based on knowledge of the empirical distribution of returns (e.g. Kahneman and Riepe [16]). Moreover, many traders use past prices as a guide to the evolution of the price in the future, with strategies using the crossing of short-run and long-run price averages being very popular (e.g. Neftci [19]). Stochastic models of markets in continuous-time in which agents use past prices to determine their demand, but in which traders discount past returns using an exponentially fading memory, include Cont and Bouchaud [6] and Hobson and Rogers [15]. In the latter paper, and in the work of Arriojas, Hu, Mohammed and Pap [3], the focus is on the pricing and hedging of options. Our work, however, is more closely related in spirit to that of Cont and Bouchaud [6], in that properties of the market model are examined.

In this paper, we present a stochastic functional differential equation model of an inefficient financial market which is a generalisation of the Black-Scholes model. The model is informationally inefficient in the sense of Fama [11], so that past movements of the stock price have an influence on future movements. The inefficiency stems from the presence of trend-following speculators, whose demand for the asset depends on the difference between a short-run and long-run weighted average of the cumulative returns on the stock over the last $\tau$ units of time. We hypothesise that speculators buy when the short-run average is above the long run average, and sell when the short-run average is below the long run average. Speculators react to other random stimuli- "news" which are independent of past returns. The increments of this news are independent, so if the stimulus is a continuous process, we may consider the returns as being driven by a one-dimensional Brownian motion $B$. Prices increase when there is excess demand, with the rise being larger the greater the excess demand, and vice versa.

This paper illustrates that there are two distinct regimes of market behaviour, which depend upon the attitudes of the investors: in one case, the cumulative returns process follows a correlated Brownian motion, while in the other, it experiences a crash or bubble. Therefore, the presence of feedback traders produces more complicated or extreme price dynamics than would be present in a corresponding efficient market model in which the driving semimartingale is a continuous Gaussian process with independent increments. A paper which considers two regimes of market behaviour, representing classical or bubblelike asymptotics, is Föllmer and Schweizer [12], where in common with this work, the dynamics depends on the strategies of market participants.

More precisely, if the trend-following speculators do not react very aggressively to differences between the short-run and long-run returns, then the rate of growth of the largest fluctuations of the solution is the same as that of a standard Brownian motion. Therefore, to a first approximation, the market appears efficient. However, the size of these largest fluctuations is greater in the presence of trend following speculators than in their absence, where the market only reacts to "news". Hence the presence of these speculators tends to increase market volatility, as well as causing correlation in the returns. The main results in this direction are part (a) of Theorem 3.1 and Theorem 3.5.

On the other hand, when the trend-following speculators behave aggressively, the returns will tend to plus or minus infinity exponentially fast, as shown by part (b) in Theorem 3.1. This is a mathematical realisation of a stock market bubble or crash. Moreover, the price history in the period before the spike in the price is of particular importance in 
determining whether a bubble or crash occurs; an upward trend in the the pre-spike period makes it more likely that a bubble occurs, while a downward trend in the prespike period makes a crash more likely. This observation is justified by Theorem 4.1.

The paper has the following structure. Section 2 gives notation and supporting results. Section 3 states the main mathematical results of the paper, while Section 4 shows how the hypotheses of these results are satisfied in the financial model. The interpretation of the results to the financial model, along with concrete examples of trading strategies, are also explored in Section 4. The model is sufficiently general so that we can capture a variety of moving average trading strategies which involve both continuous and discrete weights of past returns. The remaining sections are devoted to proofs.

\section{Preliminaries}

Let $L^{p}\left(\mathbb{R}_{+}\right), p \geqslant 1$ denote the Banach space of all Lebesgue integrable functions $f$ : $\mathbb{R}_{+} \rightarrow \mathbb{R}$ and $\mathbb{R}_{+}:=[0, \infty)$ with the standard norm, denoted by $\|\cdot\|_{L^{p}}$. The function space $C(I)$ denotes the set of all continuous functions $f: I \rightarrow \mathbb{R}$ defined on an interval $I \subseteq \mathbb{R}$ and which is equipped with the supremum norm, denoted by $\|\cdot\|_{\infty}$. The space $M(I)$ denotes the space of all signed measures on the Borel $\sigma$-algebra $\mathcal{B}(I)$ for a subset $I \subseteq \mathbb{R}$.

We first turn our attention to the deterministic delay equation which underlies the stochastic differential equation we later introduce. For a fixed constant $\tau \geq 0$ we consider the deterministic linear delay differential equation

$$
\begin{aligned}
y^{\prime}(t) & =\int_{[-\tau, 0]} y(t+u) \nu(d u) \quad \text { for } t \geq 0, \\
y(t) & =\varphi(t) \quad \text { for } t \in[-\tau, 0],
\end{aligned}
$$

for a signed measure $\nu \in M[-\tau, 0]$. The initial function $\varphi$ is assumed to be in the space $C[-\tau, 0]$. A function $y:[-\tau, \infty) \rightarrow \mathbb{R}$ is called a solution of (2.1) if $y$ is continuous on $[-\tau, \infty)$, its restriction to $[0, \infty)$ is continuously differentiable, and $y$ satisfies the first and second identity of (2.1) for all $t \geq 0$ and $t \in[-\tau, 0]$, respectively. Moreover, for every $\varphi \in C[-\tau, 0]$ the problem $(2.1)$ admits a unique solution $y(\cdot)=y(\cdot, \varphi)$.

The fundamental solution or resolvent of (2.1) is the unique locally absolutely continuous function $r:[0, \infty) \rightarrow \mathbb{R}$ which satisfies

$$
r(t)=1+\int_{0}^{t} \int_{[\max \{-\tau,-s\}, 0]} r(s+u) \nu(d u) d s \text { for all } t \geq 0 .
$$

It plays a role which is analogous to the fundamental system in linear ordinary differential equations and the Green function in partial differential equations. Formally, it is the solution of (2.1) corresponding to the initial function $\varphi=\mathbb{1}_{\{0\}}$. For later convenience we set $r(t)=0$ for $t \in(-\infty, 0)$.

The solution $y(\cdot, \varphi)$ of $(2.1)$ for an arbitrary initial segment $\varphi$ exists, is unique, and can be represented as

$$
y(t, \varphi)=\varphi(0) r(t)+\int_{[-\tau, 0]} \int_{s}^{0} r(t+s-u) \varphi(u) d u \nu(d s) \quad \text { for } t \geq 0,
$$

cf. Diekmann et al [10, Chapter I]. 
Define the function $h: \mathbb{C} \rightarrow \mathbb{C}$ by

$$
h(\lambda)=\lambda-\int_{[-\tau, 0]} e^{\lambda s} \nu(d s),
$$

and the set of its zeros

$$
\Lambda:=\{\lambda \in \mathbb{C}: h(\lambda)=0\} .
$$

The function $h$ is analytic, and so the elements of $\Lambda$ are isolated. Define

$$
v_{0}:=\sup \{\operatorname{Re}(\lambda): h(\lambda)=0\},
$$

where $\operatorname{Re}(z)$ denotes the real part of a complex number $z$. Furthermore, the cardinality of $\Lambda^{\prime}:=\Lambda \cap\left\{\operatorname{Re}(\lambda)=v_{0}\right\}$ is finite. There exists $\varepsilon_{0}>0$ such that for every $\varepsilon \in\left(0, \varepsilon_{0}\right)$ we have

$$
r(t) e^{-v_{0} t}=\sum_{\lambda_{j} \in \Lambda^{\prime}}\left(p_{j}(t) \cos \left(\operatorname{Im}\left(\lambda_{j}\right) t\right)+q_{j}(t) \sin \left(\operatorname{Im}\left(\lambda_{j}\right) t\right)\right)+o\left(e^{-\varepsilon t}\right), \quad t \rightarrow \infty,
$$

where $p_{j}$ and $q_{j}$ are polynomials of degree $m_{j}-1$, with $m_{j}$ being the multiplicity of the zero $\lambda_{j} \in \Lambda^{\prime}$ of $h$, and $\operatorname{Im}(z)$ denoting the imaginary part of a complex number $z$. This is a restatement of Diekmann et al [10, Thm. 5.4].

Let us introduce equivalent notation for (2.1). For a function $y:[-\tau, \infty) \rightarrow \mathbb{R}$ we define the segment of $y$ at time $t \geq 0$ by the function

$$
y_{t}:[-\tau, 0] \rightarrow \mathbb{R}, \quad y_{t}(u):=y(t+u) .
$$

Riesz' representation theorem guarantees that every continuous linear functional $L$ : $C[-\tau, 0] \rightarrow \mathbb{R}$ is of the form

$$
L(\psi)=\int_{[-\tau, 0]} \psi(u) \nu(d u)
$$

for a signed measure $\nu \in M[-\tau, 0]$. Hence, we will write (2.1) in the form

$$
y^{\prime}(t)=L\left(y_{t}\right) \quad \text { for } t \geq 0, \quad y_{0}=\varphi
$$

and assume $L$ to be a continuous linear functional on $C[-\tau, 0]$.

Let us fix a complete probability space $(\Omega, \mathcal{F}, P)$ with a filtration $(\mathcal{F}(t))_{t \geq 0}$ satisfying the usual conditions and let $(B(t): t \geq 0)$ be a standard Brownian motion on this space. We study the following stochastic differential equation with time delay:

$$
\begin{aligned}
d Y(t) & =L\left(Y_{t}\right) d t+\sigma d B(t) \quad \text { for } t \geq 0, \\
Y(t) & =\varphi(t) \quad \text { for } t \in[-\tau, 0],
\end{aligned}
$$

where $L$ is a continuous linear functional on $C[-\tau, 0]$ for a constant $\tau \geq 0$ and $\sigma \geqslant 0$.

For every $\varphi \in C[-\tau, 0]$ there exists a unique, adapted strong solution $(Y(t, \varphi): t \geqslant-\tau)$ with finite second moments of (2.5) (e.g. Mao [18]). The dependence of the solutions on the initial condition $\varphi$ is neglected in our notation in what follows; that is, we will write $y(t)=y(t, \varphi)$ and $Y(t)=Y(t, \varphi)$ for the solutions of (2.1) and (2.5), respectively.

By Reiß et al [22, Lemma 6.1] the solution $(Y(t): t \geq-\tau)$ of (2.5) obeys a variation of constants formula

$$
Y(t)= \begin{cases}y(t)+\int_{0}^{t} r(t-s) \sigma d B(s), & t \geq 0, \\ \varphi(t), & t \in[-\tau, 0],\end{cases}
$$

where $r$ is the fundamental solution of (2.1). 


\section{Main Theorems}

If we assume that there is only one $\lambda \in \mathbb{C}$ with $\operatorname{Re}(\lambda)=v_{0}$, i.e. $\Lambda^{\prime}=\{\lambda\}$ then it follows that $\lambda$ is real valued. If we assume furthermore that $\lambda$ is a simple zero of $h$ the representation (2.4) implies that there exists $\varepsilon_{0}>0$ such that

$$
r(t) e^{-v_{0} t}=c+o\left(e^{-\varepsilon t}\right) \quad \text { for all } \varepsilon \in\left(0, \varepsilon_{0}\right),
$$

and moreover $c$ obeys

$$
c=\frac{1}{1-\int_{[-\tau, 0]} s e^{v_{0} s} \nu(d s)} .
$$

The formula for $c$ can be determined by contour integration; see Gripenberg et al. [14, Ch.7]. The assumption that $\lambda$ is a simple zero of $h$ guarantees that $c$ is well defined because the denumerator of $c$ equals $h^{\prime}(\lambda)$, i.e. is non-zero.

Theorem 3.1. Suppose that $r$ obeys (3.1). Then the solution $Y$ of (2.5) satisfies

(a) if $v_{0}=0$ and $r^{\prime} \in L^{2}\left(\mathbb{R}_{+}\right)$, then a.s.

$$
-\sigma|c|=\liminf _{t \rightarrow \infty} \frac{Y(t)}{\sqrt{2 t \log \log t}} \leqslant \limsup _{t \rightarrow \infty} \frac{Y(t)}{\sqrt{2 t \log \log t}}=\sigma|c| .
$$

(b) if $v_{0}>0$, then a.s.

$$
\begin{aligned}
& \lim _{t \rightarrow \infty} e^{-v_{0} t} Y(t) \\
& \quad=c \int_{-\tau}^{0} e^{-v_{0} u}(\varphi(u)-\varphi(0))\left(\int_{[-\tau, u]} e^{v_{0} s} \nu(d s)\right) d u+c \sigma \int_{0}^{\infty} e^{-v_{0} s} d B(s) .
\end{aligned}
$$

In both cases, the constant $c$ is given by (3.2).

\section{Remark 3.2.}

(a) The case $v_{0}<0$ is discussed in [13], with pathwise asymptotic behaviour being considered in [5]. It turns out in this case that all solutions converge weakly to a stationary distribution. In our market model this situation does not occur and will therefore be not considered.

(b) Contrary to classical research on the Liapunov spectrum of affine stochastic functional equations, part (b) of Theorem 3.1 quantifies not only the leading Liapunov exponent, but also the corresponding random multiplier.

(c) The stochastic integral with unbounded domain in part (b) of Theorem 3.1 is defined as the limit in mean square of the random variables

$$
W(T):=\int_{0}^{T} e^{-v_{0} s} d B(s)
$$

for $T \rightarrow \infty$. By the martingale convergence theorem, the convergence also takes place a.s. 
The random variable $\Gamma(\varphi)$, defined by the right hand side in (b) of Theorem 3.1,

$$
\Gamma(\varphi):=c \int_{-\tau}^{0} e^{-v_{0} u}(\varphi(u)-\varphi(0))\left(\int_{[-\tau, u]} e^{v_{0} s} \nu(d s)\right) d u+c \sigma \int_{0}^{\infty} e^{-v_{0} s} d B(s)
$$

is normally distributed with

$$
\begin{aligned}
E[\Gamma(\varphi)] & =c \int_{-\tau}^{0} e^{-\lambda u}(\varphi(u)-\varphi(0))\left(\int_{[-\tau, u]} e^{v_{0} s} \nu(d s)\right) d u \\
\operatorname{Var}[\Gamma(\varphi)] & =(c \sigma)^{2} \int_{0}^{\infty} e^{-2 v_{0} s} d s .
\end{aligned}
$$

Thus, if $\sigma \neq 0$ there is always a positive probability that the limit is positive, and a positive probability that the limit is negative.

Given a measure $\nu$ it is often a rather delicate issue to determine the value of the leading zero of $h$, or even decide between the two cases considered in Theorem 3.1. In the following result, a simple criterion is given for a subclass of all signed measures in $M[-\tau, 0]$ which will later cover the economic modelling.

Theorem 3.3. Suppose that $0 \neq \nu \in M[-\tau, 0]$ obeys

$$
\begin{aligned}
& \nu([-t, 0]) \geq 0 \quad \text { for all } t \in[0, \tau] \\
& \nu([-\tau, 0])=0
\end{aligned}
$$

(a) If

$$
m(\nu):=\int_{[-\tau, 0]} s \nu(d s)>1,
$$

then $h$ has a simple zero at $\lambda=v_{0}>0$ and all other zeros $\lambda$ of $h$ obey $\operatorname{Re}(\lambda)<v_{0}$.

(b) If

$$
m(\nu):=\int_{[-\tau, 0]} s \nu(d s)<1,
$$

then $h$ has a simple zero at $\lambda=v_{0}=0$ and all other zeros $\lambda$ of $h$ obey $\operatorname{Re}(\lambda)<v_{0}$.

\section{Remark 3.4.}

(a) In the proof of Theorem 3.3 we derive in both cases that $h^{\prime}\left(v_{0}\right)>0$, which implies that the constant $c$ defined in (3.2) is always positive.

(b) The conditions (3.5) and (3.6) also yield that $m(v) \geqslant 0$, for

$$
m(v)=\int_{[-\tau, 0]} \int_{-\tau}^{s} d t \nu(d s)=\int_{-\tau}^{0} \int_{[t, 0]} \nu(d s) d t \geqslant 0 .
$$

(c) The conditions (3.5) and (3.6) together with $m(\nu)<1$ imply that $r^{\prime} \in L^{p}\left(\mathbb{R}_{+}\right)$for every $p \geqslant 1$, see Lemma 7.1. Thus, under these conditions, all requirements in part (a) of Theorem 3.1 are satisfied. 
In economic modelling the $\delta$-return provides information on the percentage gains or losses made by investing over a time period of $\delta$ units. In our economic modelling in the next section the $\delta$-returns are given by the process $\left(Y_{\delta}(t): t \geqslant \delta\right)$ for a fixed constant $\delta>0$ defined by

$$
Y_{\delta}(t):=Y(t)-Y(t-\delta) \quad \text { for all } t \geqslant \delta .
$$

Recall that we set $r(t)=0$ for $t \in(-\infty, 0)$ and let us introduce the function $r_{\delta}$ defined by

$$
r_{\delta}(t):=r(t)-r(t-\delta) \quad \text { for all } t \geq 0 .
$$

The variation of constants formula (2.6) implies the identity

$$
Y_{\delta}(t)=y(t)-y(t-\delta)+\int_{0}^{t} r_{\delta}(t-s) \sigma d B(s) \quad \text { for all } t \geq \delta
$$

The next theorem determines the asymptotic behaviour of the autocorrelation function of $Y_{\delta}$. We will later interpret these results in our economic setting.

Theorem 3.5. Suppose that $0 \neq \nu \in M[-\tau, 0]$ obeys (3.5) and (3.6) and $m(\nu)<1$. For $\delta>0$ and $\Delta \geq 0$ we have that

(a) $\operatorname{Cov}\left(Y_{\delta}(t), Y_{\delta}(t+\Delta)\right)>0$ for all $t \geq \delta$.

(b) The limit

$$
c_{\delta}(\Delta):=\lim _{t \rightarrow \infty} \operatorname{Cov}\left(Y_{\delta}(t), Y_{\delta}(t+\Delta)\right)=\sigma^{2} \int_{0}^{\infty} r_{\delta}(u) r_{\delta}(u+\Delta) d u
$$

exists and is finite. Moreover, for each $\delta>0$ we have

$$
\lim _{\Delta \rightarrow \infty} c_{\delta}(\Delta)=0 \quad \text { and } \quad c_{\delta} \in L^{1}\left(\mathbb{R}_{+}\right) .
$$

(c) There exists a unique $\lambda_{0}>0$ such that $-\lambda_{0} \in \Lambda$ and

$$
\lim _{\Delta \rightarrow \infty} c_{\delta}(\Delta) e^{\lambda_{0} \Delta}=\sigma^{2}\left(\int_{0}^{\tau} u e^{\lambda_{0} u} \nu([-u, 0]) d u\right)^{-1}\left(\int_{0}^{\delta} e^{\lambda_{0} u} d u\right)\left(\int_{0}^{\infty} r_{\delta}(u) e^{-\lambda_{0} u} d u\right)
$$

with the limit being finite and positive.

In $[1,2]$ the asymptotic behaviour of the autocovariance function of the solution of a linear stochastic integral equation is studied, and criteria for long memory established. In common with our work, it is found that the asymptotic behaviour of the autocovariance function depends on the asymptotic behaviour of the fundamental solution of an underlying deterministic linear functional equation.

\section{Applications to Financial Markets}

\subsection{Economic modelling}

We now consider equation (2.5) in the context of a market model. Let $(S(t): t \geqslant 0)$ denote the prices of a risky asset which satisfy

$$
d S(t)=S(t) d R(t) \quad \text { for all } t \geqslant 0,
$$


where $R(t)$ denotes the cumulative return at time $t$. We assume that the cumulative returns $R$ follow a linear trend $\mu$. Suppose that there are $N$ traders in the economy, who determine their demand based on the cumulative de-trended returns $Y(t):=R(t)-\mu t$ on the asset. The trading strategy of the $j$-th agent at time $t$ is as follows: they consider a short-run moving average of the cumulative de-trended returns price over the last $\vartheta_{j}$ units of time

$$
\int_{\left[-\vartheta_{j}, 0\right]} Y(t+u) s_{j}(d u)
$$

for a signed measure $s_{j} \in M\left[-\vartheta_{j}, 0\right]$ and also calculate a long-run average of cumulative de-trended returns over the last $\tau_{j} \geqslant \vartheta_{j}$ units of time

$$
\int_{\left[-\tau_{j}, 0\right]} Y(t+u) l_{j}(d u)
$$

for a signed measure $l_{j} \in M\left[-\tau_{j}, 0\right]$. The measures $s_{j}$ and $l_{j}$ reflect the weights the agent puts on the different past values. In order to make the short-run and long-run comparable the measures $s_{j}$ and $l_{j}$ are chosen such that

$$
s_{j}\left(\left[-\vartheta_{j}, 0\right]\right)=l_{j}\left(\left[-\tau_{j}, 0\right]\right)
$$

We extend $s_{j}$ to $M\left[-\tau_{j}, 0\right]$ by setting $s_{j}(I)=0$ for any Borel set $I \subseteq\left[-\tau_{j}, \vartheta_{j}\right)$. These averages can be distinguished as being "short-run" and "long-run" by hypothesising that the short-run average always allocates at least as much weight to the most recent $t$ time units of returns as the long-run average does. Mathematically, this means that

$$
s_{j}([-t, 0]) \geqslant l_{j}([-t, 0]) \quad \text { for all } t \in\left[0, \tau_{j},\right] .
$$

The averages are distinguishable by presuming that

$$
s_{j} \neq l_{j}
$$

Trader $j$ then has planned demand at time $t$ which depends upon the strength of the signal received from the market, the signal being stronger the greater the difference between the short-run and long run-average. We assume in the sequel that the trader buys the asset if the short-run average exceeds the long-run average and that they sell the asset if the short-run average lies below the long run average. The planned excess demand of trader $j$ at time $t$ is

$$
\beta_{j}\left(\int_{\left[-\vartheta_{j}, 0\right]} Y(t+u) s_{j}(d u)-\int_{\left[-\tau_{j}, 0\right]} Y(t+u) l_{j}(d u)\right)
$$

where $\beta_{j} \geqslant 0$. Therefore, the overall planned excess demand of all $N$ traders is

$$
\sum_{j=1}^{N} \beta_{j}\left(\int_{\left[-\vartheta_{j}, 0\right]} Y(t+u) s_{j}(d u)-\int_{\left[-\tau_{j}, 0\right]} Y(t+u) l_{j}(d u)\right) .
$$

The constants $\beta_{j}$ model the different influence of each trader on the total excess demand. Speculators react to other random stimuli - "news" - which are independent of past returns. The increments of this news are independent, so if the stimulus is a continuous process, this may be thought of as adding a further $\sigma\left(B\left(t_{2}\right)-B\left(t_{1}\right)\right)$ to the traders' 
excess demand over the interval $\left[t_{1}, t_{2}\right]$ where $B$ is a one-dimensional Brownian motion and $\sigma \geqslant 0$.

Finally, we suppose that the de-trended returns increase when there is excess demand, with the rise being larger the greater the excess demand. One way to capture this is to suppose that the evolution of the de-trended returns is described by

$$
d Y(t)=\sum_{j=1}^{N} \beta_{j}\left(\int_{\left[-\vartheta_{j}, 0\right]} Y(t+u) s_{j}(d u)-\int_{\left[-\tau_{j}, 0\right]} Y(t+u) l_{j}(d u)\right) d t+\sigma d B(t) .
$$

We extend all measures $s_{j}$ and $l_{j}$ to the interval $[-\tau, 0]$ where $\tau=\max \left\{\tau_{1}, \ldots, \tau_{N}\right\}$ by setting them to zero outside their support. By introducing the measure $\nu \in M[-\tau, 0]$ defined by

$$
\nu(d u):=\sum_{j=1}^{N} \beta_{j}\left(s_{j}-l_{j}\right)(d u)
$$

and the linear functional $L$ defined by

$$
L: C[-\tau, 0] \rightarrow \mathbb{R}, \quad L \varphi=\int_{[-\tau, 0]} \varphi(u) \nu(d u)
$$

we can rewrite equation (4.5) as

$$
d Y(t)=L\left(Y_{t}\right) d t+\sigma d B(t) \quad \text { for all } t \geqslant 0 .
$$

Note that under the conditions (4.2), (4.3) and (4.4) on the measures $s_{j}$ and $l_{j}$, the measure $\nu$ satisfies the conditions in Theorem 3.3.

Because of (4.1), the evolution of the price of the risky asset $(S(t): t \geq 0)$ is now given by

$$
d S(t)=\mu S(t) d t+S(t) d Y(t), \quad t \geq 0 .
$$

Applying Itô's formula shows as in the standard Black-Scholes model that the asset price $S$ can be represented by

$$
S(t)=S(0) \exp \left(Y(t)+\left(\mu-\frac{1}{2} \sigma^{2}\right) t\right) \quad \text { for all } t \geqslant 0 .
$$

In the case when the feedback traders are absent, i.e. $\beta_{j}=0$ for all $j=1, \ldots, N$, we have $d Y(t)=\sigma d B(t)$, in which case $S$ is Geometric Brownian motion, evolving according to

$$
d S(t)=\mu S(t) d t+\sigma S(t) d B(t) \quad \text { for all } t \geqslant 0 .
$$

In this case our model coincides with the standard Black-Scholes model and can be considered as a generalisation of it.

\subsection{Economic interpretation of main results}

Before considering specific examples of moving average strategies of the traders, we make some general comments about the economic implications of these results. 


\subsubsection{Dynamical behavior of the market}

If $m(\nu)<1$, by combining Theorem 3.1, Theorem 3.3 and Remark 3.4.(c), we have

$$
\limsup _{t \rightarrow \infty} \frac{Y(t)}{\sqrt{2 t \log \log t}}=\frac{\sigma}{1-m(\nu)} \text { a.s., }
$$

which, because of (4.7), yields

$$
\limsup _{t \rightarrow \infty} \frac{\log S(t)-\left(\mu-\frac{1}{2} \sigma^{2}\right) t}{\sqrt{2 t \log \log t}}=\frac{\sigma}{1-m(\nu)} \quad \text { a.s. }
$$

with a similar result available for the limit inferior. Under the two conditions (3.5) and (3.6) which we assume throughout this section, we have $m(\nu) \geq 0$ according to Remark 3.4 , and hence the limit on the right hand side satisfies

$$
\frac{\sigma}{1-m(\nu)} \in[\sigma, \infty)
$$

Therefore, the process $S$ experiences larger fluctuations the closer the value $m(\nu)$ is to 1. On the other hand, $m(\nu)=0$ occurs in the absence of the trend chasing speculators. In this case, or with the value $m(\nu)$ close to zero, the fluctuations are of a similar size as in the standard Black-Scholes model. In other words, the presence of the trend chasing speculators makes the market more risky, and leads to greater fluctuations. This is similar to findings of DeLong, Shleifer, Summers and Waldman [9], in which the presence of noise traders increases the risk for informed investors.

If $m(\nu)>1$ then combining Theorem 3.1 and Theorem 3.3 implies

$$
\lim _{t \rightarrow \infty} e^{-v_{0} t} Y(t)=\Gamma(\varphi) \quad \text { a.s. }
$$

where $\Gamma(\varphi)$ is the normally distributed random variable defined in (3.3). In this situation we have

$$
\lim _{t \rightarrow \infty} \frac{\log S(t)-\left(\mu-\frac{1}{2} \sigma^{2}\right) t}{\exp \left(v_{0} t\right)}=\Gamma(\varphi) \quad \text { a.s. }
$$

Since the random variable $\Gamma(\varphi)$ is normally distributed there is a non-zero probability that $\log S(t)-\left(\mu-\frac{1}{2} \sigma^{2}\right) t$ converges a.s. exponentially to $\infty$, representing a bubble, or to $-\infty$, representing a crash. Of course, the probabilities of these events depend on the expectation of the random variable $\Gamma(\varphi)$, which will be the objective of the following subsection.

In both cases the fluctuations increase in size with $m(\nu)$. We now investigate the factors that increase this quantity, to which end we define

$$
\begin{aligned}
m(\nu)=\int_{[-\tau, 0]} u \nu(d u) & =\sum_{j=1}^{N} \beta_{j}\left(\int_{\left[-\vartheta_{j}, 0\right]} u s_{j}(d u)-\int_{\left[-\tau_{j}, 0\right]} u l_{j}(d u)\right) \\
& =: \sum_{j=1}^{N} \beta_{j}\left(m\left(s_{j}\right)-m\left(l_{j}\right)\right) .
\end{aligned}
$$

The quantity $m\left(s_{j}\right)$ indicates the weight that trader $j$ gives to recent returns when computing their short-run moving average and similarly for $m\left(l_{j}\right)$. The greater the 
difference $m\left(s_{j}\right)-m\left(l_{j}\right)$ between these values, the larger the value of $m(\nu)$, and the more unstable the market becomes. It may be seen that a large value of $m\left(s_{j}\right)-m\left(l_{j}\right)$ arises, for example, when trader $j$ bases their short-run average on returns over a very short time-horizon, but whose long-run average gives significant weight to returns from the relatively distant past. This strategy can obviously introduce significant feedback from the distant past, so causing trends from the returns in the past to persist for long periods of time, which will tend to cause excess volatility. More specific examples will be considered in Section 4.3.

A large value of $\beta_{j}$ corresponds to aggressive or confident speculative behaviour. The planned excess demand of trader $j$ is $\beta_{j}$ times the difference between the short-run and long-run weighted averages of returns. Therefore, for larger $\beta_{j}$, a smaller signal from the market is required to produce a given response from trader $j$.

We see that aggressive responses from traders and giving significant weight to the returns in the more distant past will tend to destabilise the market. In fact, when these effects are so pronounced that $m(\nu)>1$ we have that $\lim _{t \rightarrow \infty} e^{-v_{0} t} Y(t)=: \Gamma(\varphi)$ exists, is almost surely non-zero, and attains positive and negative values with positive probability, which correspond to a bubble or crash.

\subsubsection{Bubble and Crash Dynamics}

In this subsection, we assume $m(\nu)>1$, and consider the probability of a crash or bubble in terms of the initial, or pre-spike returns $\varphi$. The following results follow immediately from the expectation (3.4) of the random variable $\Gamma(\varphi)$.

(a) If $\varphi(u)=k$ for all $u \in[-\tau, 0]$ and for a constant $k \in \mathbb{R}$ then $E[\Gamma(\varphi)]=0$ and therefore

$$
P(\Gamma(\varphi)<0)=P(\Gamma(\varphi)>0)=\frac{1}{2} .
$$

Thus, if there is no trend in the returns on $[-\tau, 0]$, then the market is equally likely to enter a bubble or a crash. This is sensible because the traders are not able to detect a trend in the market which might influence their decisions in one direction or another.

(b) If two pre-spike sets of returns are shifted, so that $\varphi_{2}(u)=\varphi_{1}(u)+k$ for a constant $k \in \mathbb{R}$, it follows that

$$
P\left(\Gamma\left(\varphi_{1}\right)>0\right)=P\left(\Gamma\left(\varphi_{2}\right)>0\right) .
$$

This suggests that the patterns of the recent returns influences the probability of a bubble rather than whether the returns are high or low.

(c) If $P(\Gamma(\varphi)>0)>\frac{1}{2}$ then

$$
\begin{aligned}
& \alpha \mapsto P(\Gamma(\alpha \varphi)>0) \quad \text { is increasing, } \\
& \lim _{\alpha \rightarrow \infty} P(\Gamma(\alpha \varphi)>0)=1, \quad \lim _{\alpha \rightarrow-\infty} P(\Gamma(\alpha \varphi)>0)=0 .
\end{aligned}
$$

This suggests that if there is a trend in the initial returns which makes the probability of a bubble more likely than that of a crash, an amplified version of that trend would make a bubble even more likely to occur, with greater amplifying factors leading to greater probabilities of a bubble. This suggests that when the traders receive stronger trending signals from the market, they are more likely to make these trends self-fulfilling. 
(d) The following theorem considers how an increasing trend in the initial returns can lead to speculators extrapolating this rising trend, thus making a bubble more likely.

Theorem 4.1. Suppose that $0 \neq \nu \in M[-\tau, 0]$ obeys (3.5) and (3.6) and $m(\nu)>1$. For an initial return $\varphi \in C^{1}([-\tau, 0])$ with $\varphi^{\prime}(0) \neq 0$ we have that:

(a) if $\varphi$ is increasing then $P(\Gamma(\varphi)>0)>\frac{1}{2}$;

(b) if $\varphi$ is decreasing then $P(\Gamma(\varphi)<0)>\frac{1}{2}$.

The properties (a)-(d) above concentrate on the impact of the initial returns on the probability of a bubble or crash. However, this probability also depends on the properties of the Itô integral on the righthand side of (3.3). Since the integrand of the Itô integral on the righthand side of (3.3) decreases with time, the impact of "news" at the outset is significant for the evolution of the spike, with initial good news about the stock tending to result in a positive value of the Itô integral. Therefore, if there is good initial news about the asset, the price of the stock tends to increase, and the traders force the price higher by misperceiving this increase as arising from demand from informed speculators. As before, this induces further buying, and the stock price undergoes a bubble.

These remarks suggest that the mechanisms by which bubbles form in this model are consistent with the notion of mimetic contagion introduced by Orléan [20]. In mimetic contagion, we may think of the market as comprising of two forms of traders, with new entrants choosing the trading strategy which tends to dominate at a given time. In the long-run, the proportion of traders in each category settles down to a value which is random but which depends quite strongly on what happens in the first trading periods. The similarities with mimetic contagion are as follows: in (3.3), the righthand side depends crucially on the market behaviour at the outset; once a dominant trend becomes apparent, the trend following speculators will tend to extrapolate that trend; and the longrun behaviour (either a bubble or crash) is not known in advance.

\subsubsection{Autocovariance}

In this subsection, we analyse the patterns in the $\delta$-returns when the measure $\nu$ obeys (3.5), (3.6) and the stability condition $m(\nu)<1$ holds. Since the $\delta$-returns are simply the percentage gains or losses made by investing over a time period of $\delta$ units they are given by the process $Y_{\delta}$ defined in (3.7) by $Y_{\delta}(t):=Y(t)-Y(t-\delta)$ for $t \geqslant \delta$. Theorem 3.5 states that the $\delta$-returns are positively autocorrelated. Therefore, even though the returns undergo iterated logarithm behavior like standard Brownian motion, there is correlation between the increments of the process. The presence of a positive correlation means that trends in the returns have a tendency to persist. This is responsible for the fact that the largest fluctuations of the process $Y$ are greater than those that would be seen if there were no trend-following speculators present. The correlation between returns of horizon length $\delta$ decays exponentially in the time lag $\Delta$ between successive observations, as $\Delta \rightarrow \infty$. Moreover, the exponent in the rate of decay is independent of $\Delta$. Therefore, although the market is informationally inefficient because the future returns are correlated with past returns, the memory of recent events is discounted relatively quickly. This short memory is a consequence of the finite memory trading strategies employed by agents. 


\subsection{Examples of investment strategies}

In each of the following examples, we consider only one agent and their trading strategy. Because of this we neglect the parameter $\beta$ in the model which weighs only the influence of a single investor on the total cumulative returns.

\subsubsection{Current returns versus past returns}

Suppose that the investor compares the current value of the cumulative returns $Y$ with a continuous time weighted average over the last $\tau$ units. To put this in the form of the model considered in Section 4.1, the current value of the cumulative returns is weighted by

$$
s(d u)=\alpha \delta_{0}(d u)
$$

for a constant $\alpha>0$, where $\delta_{0}$ denotes the Dirac measure in 0 . The cumulative returns in the long-run are weighted by

$$
l(d u)=f(u) d u,
$$

where $f$ is a nonnegative function in $L^{1}[-\tau, 0]$ with $\|f\|_{L^{1}}=\alpha$ for some $\tau>0$. Then the measure

$$
\nu(d u):=s(d u)-l(d u)=\alpha \delta_{0}(d u)-f(u) d u
$$

satisfies the conditions in Theorem 3.3 with the moment given by

$$
m(\nu)=-\int_{-\tau}^{0} s f(s) d s
$$

The linear functional $L$ is of the form

$$
L: C[-\tau, 0] \rightarrow \mathbb{R}, \quad L(\varphi)=\alpha \varphi(0)-\int_{-\tau}^{0} \varphi(s) f(s) d s .
$$

If $m(\nu)<1$ then the cumulative returns obey

$$
\begin{aligned}
& \limsup _{t \rightarrow \infty} \frac{Y(t)}{\sqrt{2 t \log \log t}}=\frac{|\sigma|}{1-m(\nu)} \quad \text { a.s. } \\
& \liminf _{t \rightarrow \infty} \frac{Y(t)}{\sqrt{2 t \log \log t}}=\frac{-|\sigma|}{1-m(\nu)} \quad \text { a.s. }
\end{aligned}
$$

On the other, hand if $m(\nu)>1$ then there exists a unique $\lambda>0$ such that a.s.

$$
\begin{aligned}
& \lim _{t \rightarrow \infty} e^{-\lambda t} Y(t) \\
& =\frac{1}{1+\int_{-\tau}^{0} s e^{\lambda s} f(s) d s}\left(\int_{-\tau}^{0}(\varphi(0)-\varphi(u)) \int_{-\tau}^{u} e^{\lambda(s-u)} f(s) d s d u+\sigma \int_{0}^{\infty} e^{-\lambda s} d B(s)\right) .
\end{aligned}
$$




\subsubsection{Short run versus long run moving averages}

Suppose that the investor compares a continuous time weighted average of the cumulative returns $Y$ over the last $\vartheta$ units of time with a moving average over the last $\tau \geqslant \vartheta$ units of time. The short-run is weighted by a nonnegative function $f \in L^{1}[-\vartheta, 0]$ while the long-run by a nonnegative function $g \in L^{1}[-\tau, 0]$ with $\|f\|_{L^{1}}=\|g\|_{L^{1}}>0$. We extend $f$ to $[-\tau, 0]$ by setting $f(u)=0$ for $u \in[-\tau,-\vartheta)$. If we suppose in addition that

$$
\int_{-t}^{0} f(s) d s \geq \int_{-t}^{0} g(s) d s \quad \text { for all } t \in[-\tau, 0],
$$

then the measure $\nu(d u):=(f(u)-g(u)) d u$ satisfies all the conditions in Theorem 3.3 with the moment

$$
m(\nu)=\int_{-\tau}^{0} s(f(s)-g(s)) d s .
$$

The linear functional $L$ is given by

$$
L: C[-\tau, 0] \rightarrow \mathbb{R}, \quad L(\varphi)=\int_{-\tau}^{0} \varphi(s)(f(s)-g(s)) d s .
$$

According to Theorem 3.1 if we have $m(\nu)<1$ then the cumulative returns obey

$$
\begin{aligned}
& \limsup _{t \rightarrow \infty} \frac{Y(t)}{\sqrt{2 t \log \log t}}=\frac{|\sigma|}{1-m(\nu)} \quad \text { a.s., } \\
& \liminf _{t \rightarrow \infty} \frac{Y(t)}{\sqrt{2 t \log \log t}}=\frac{-|\sigma|}{1-m(\nu)} \quad \text { a.s. }
\end{aligned}
$$

On the other hand, if $m(\nu)>1$ then there exists a unique positive $\lambda>0$ such that

$$
\begin{aligned}
& \lim _{t \rightarrow \infty} e^{-\lambda t} Y(t) \\
& =c\left(\int_{-\tau}^{0}(\varphi(0)-\varphi(u)) \int_{-\tau}^{u} e^{\lambda(s-u)}(f(s)-g(s)) d s d u+\sigma \int_{0}^{\infty} e^{-\lambda s} d B(s)\right),
\end{aligned}
$$

where $c=\left(1-\int_{-\tau}^{0} s e^{\lambda s}(f(s)-g(s)) d s\right)^{-1}$.

\subsubsection{Discrete-time moving averages}

Suppose that the investor compares a weighted average of the cumulative returns at $m$ points in time over the last $\vartheta$ units of time with a weighted average of the cumulative returns at $n$ points in time over the last $\tau$ units of time, where $\tau \geqslant \vartheta$. Let the cumulative returns in the short-run be observed at time points $-\vartheta=-\vartheta_{1}<\cdots<-\vartheta_{m}=0$ and in the long-run at time points $-\tau=-\tau_{1}<\cdots<-\tau_{n}=0$. Then the short-run observations are averaged according to a measure

$$
s(d u)=\sum_{j=1}^{m} \alpha_{j} \delta_{-\vartheta_{j}}(d u)
$$


for some weights $\alpha_{j} \geqslant 0$ and the long-run observations according to

$$
l(d u)=\sum_{j=1}^{n} \beta_{j} \delta_{-\tau_{j}}(d u)
$$

for some weights $\beta_{j} \geqslant 0$. If we assume that

$$
\begin{aligned}
\alpha_{1}+\cdots+\alpha_{m} & =\beta_{1}+\cdots+\beta_{n}>0, \\
\sum_{j=1}^{m} \alpha_{j} \mathbb{1}_{[-t, 0]}\left(-\vartheta_{j}\right) & \geqslant \sum_{j=1}^{n} \beta_{j} \mathbb{1}_{[-t, 0]}\left(-\tau_{j}\right) \quad \text { for all } t \in[0, \tau],
\end{aligned}
$$

then the measure $\nu(d u):=s(d u)-l(d u)$ satisfies all the conditions in Theorem 3.3 with the moment

$$
m(\nu)=\sum_{j=1}^{n} \beta_{j} \tau_{j}-\sum_{j=1}^{m} \alpha_{j} \vartheta_{j} .
$$

The linear functional $L$ is given by

$$
L: C[-\tau, 0] \rightarrow \mathbb{R}, \quad L(\varphi)=\sum_{j=1}^{m} \alpha_{j} \varphi\left(-\vartheta_{j}\right)-\sum_{j=1}^{n} \beta_{j} \varphi\left(-\tau_{j}\right) .
$$

If $m(\nu)<1$ then the cumulative returns evolve according to

$$
\begin{aligned}
& \limsup _{t \rightarrow \infty} \frac{Y(t)}{\sqrt{2 t \log \log t}}=\frac{|\sigma|}{1-m(\nu)} \quad \text { a.s., } \\
& \liminf _{t \rightarrow \infty} \frac{Y(t)}{\sqrt{2 t \log \log t}}=\frac{-|\sigma|}{1-m(\nu)} \quad \text { a.s. }
\end{aligned}
$$

On the other hand, if $m(\nu)>1$ then there exists a unique positive $\lambda>0$ such that a.s.

$$
\begin{aligned}
& \lim _{t \rightarrow \infty} e^{-\lambda t} Y(t)=c \sigma \int_{0}^{\infty} e^{-\lambda s} d B(s) \\
& \quad+c\left(\sum_{i=1}^{m-1} \alpha_{i} e^{-\vartheta_{i} \lambda} \int_{-\vartheta_{i}}^{0} e^{-\lambda u}(\varphi(u)-\varphi(0)) d u-\sum_{j=1}^{n-1} \beta_{i} e^{-\tau_{i} \lambda} \int_{-\tau_{i}}^{0} e^{-\lambda u}(\varphi(u)-\varphi(0)) d u\right),
\end{aligned}
$$

where we assume without any restriction $m, n \geqslant 2$ and

$$
c=\left(1-\sum_{j=1}^{n} \beta_{j} \tau_{j} e^{-\lambda \tau_{j}}+\sum_{j=1}^{m} \alpha_{j} \vartheta_{j} e^{-\lambda \vartheta_{j}}\right)^{-1} .
$$

\section{$5 \quad$ Proof of Theorem 3.1}

We start this section by proving an iterated logarithm-type law for the Gaussian process $(Q(t): t \geqslant 0)$ defined by

$$
Q(t):=\int_{0}^{t} f(t-s) d B(s)
$$

for a function $f$ in the Sobolev space $W^{2,1}\left(\mathbb{R}_{+}\right)$. Semimartingale properties of the process $Q$ and other questions are considered in [7]. 
Lemma 5.1. Suppose that $f \in W^{2,1}\left(\mathbb{R}_{+}\right)$. Then the Gaussian process $Q$ defined in (5.1) satisfies

$$
\limsup _{t \rightarrow \infty} \frac{|Q(t)|}{\sqrt{2 \log t}} \leqslant\|f\|_{L^{2}} \quad \text { a.s. }
$$

Proof. Because $f$ is almost everywhere differentiable we obtain by partial integration and a stochastic Fubini Theorem (e.g. [21, Ch.IV.6, Thm.64])

$$
\begin{aligned}
Q(t) & =\int_{0}^{t}\left(f(0)+\int_{0}^{t-s} f^{\prime}(u) d u\right) d B(s) \\
& =\int_{0}^{t}\left(\int_{0}^{v} f^{\prime}(v-s) d B(s)\right) d v+f(0) B(t) .
\end{aligned}
$$

Thus, for arbitrary $\varepsilon \in(0,1)$ and $n \in \mathbb{N}$ we can decompose $Q(t)$ according to

$$
\begin{aligned}
Q(t) & =Q(t)-Q\left(n^{\varepsilon}\right)+Q\left(n^{\varepsilon}\right) \\
& =f(0)\left(B(t)-B\left(n^{\varepsilon}\right)\right)+\int_{n^{\varepsilon}}^{t}\left(\int_{0}^{v} f^{\prime}(v-s) d B(s)\right) d v+Q\left(n^{\varepsilon}\right) .
\end{aligned}
$$

We now analyse each term in (5.2). By time inversion and Lévy's modulus of continuity, the first term obeys

$$
\lim _{n \rightarrow \infty} \sup _{n^{\varepsilon} \leq t \leq(n+1)^{\varepsilon}} \frac{|f(0)|\left|B(t)-B\left(n^{\varepsilon}\right)\right|}{\sqrt{\varepsilon \log n}}=0 \quad \text { a.s. }
$$

To estimate the second term in (5.2), we start by choosing $k \in \mathbb{N}$ such that $(1-\varepsilon) 2 k>1$ and letting

$$
U_{n}:=\sup _{n^{\varepsilon} \leq t \leq(n+1)^{\varepsilon}}\left|\int_{n^{\varepsilon}}^{t}\left(\int_{0}^{v} f^{\prime}(v-s) d B(s)\right) d v\right| .
$$

Applying the Cauchy-Schwarz inequality implies

$$
\begin{aligned}
\mathbb{E}\left[U_{n}^{2 k}\right] & =E\left[\sup _{n^{\varepsilon} \leq t \leq(n+1)^{\varepsilon}}\left|\int_{n^{\varepsilon}}^{t}\left(\int_{0}^{v} f^{\prime}(v-s) d B(s)\right) d v\right|^{2 k}\right] \\
& \leq E\left[\sup _{n^{\varepsilon} \leq t \leq(n+1)^{\varepsilon}}\left(t-n^{\varepsilon}\right)^{2 k-1}\left(\int_{n^{\varepsilon}}^{t}\left|\int_{0}^{v} f^{\prime}(v-s) d B(s)\right|^{2 k} d v\right)\right] \\
& =\left((n+1)^{\varepsilon}-n^{\varepsilon}\right)^{2 k-1} \int_{n^{\varepsilon}}^{(n+1)^{\varepsilon}} E\left[J(v)^{2 k}\right] d v,
\end{aligned}
$$

where $J(v):=\int_{0}^{v} f^{\prime}(v-s) d B(s)$ is normally distributed with zero mean and variance $\int_{0}^{v} f^{\prime}(s)^{2} d s$. Since $f^{\prime} \in L^{2}\left(\mathbb{R}_{+}\right)$we have $E\left[J(v)^{2 k}\right]=c_{k}\left(\int_{0}^{v} f^{\prime}(s)^{2} d s\right)^{k} \leq c_{k}\left\|f^{\prime}\right\|_{L^{2}}^{2 k}$ for a constant $c_{k}>0$ and it follows

$$
\mathbb{E}\left[U_{n}{ }^{2 k}\right] \leqslant\left((n+1)^{\varepsilon}-n^{\varepsilon}\right)^{2 k} c_{k}\left\|f^{\prime}\right\|_{L^{2}}^{2 k} \leqslant \varepsilon^{2 k-1} n^{(\varepsilon-1) 2 k} c_{k}\left\|f^{\prime}\right\|_{L^{2}}^{2 k} .
$$

Because $(1-\varepsilon) 2 k>1$ the Borel-Cantelli Lemma implies

$$
\limsup _{n \rightarrow \infty} \sup _{n^{\varepsilon} \leqslant t \leqslant(n+1)^{\varepsilon}}\left|\int_{n^{\varepsilon}}^{t}\left(\int_{0}^{v} f^{\prime}(v-s) d B(s)\right) d v\right| \leqslant 1 \quad \text { a.s. }
$$


To estimate the last term in (5.2), we define the standardized normal random variable

$$
Z_{n^{\varepsilon}}:=Q\left(n^{\varepsilon}\right)\left(\int_{0}^{n^{\varepsilon}} f^{2}(s) d s\right)^{-1 / 2} .
$$

For any $\vartheta>1$ we get by Mill's estimate

$$
\begin{aligned}
P\left(\left|Z_{n^{\varepsilon}}\right|>\sqrt{2 \vartheta \log \left(n^{\varepsilon}\right)}\right) & \leq \frac{2}{\sqrt{2 \pi}} \cdot \frac{1}{\sqrt{2 \vartheta \log \left(n^{\varepsilon}\right)}} \cdot e^{-\vartheta \log \left(n^{\varepsilon}\right)} \\
& =\frac{2}{\sqrt{2 \pi}} \cdot \frac{1}{\sqrt{2 \vartheta \log \left(n^{\varepsilon}\right)}} \cdot \frac{1}{\left(n^{\varepsilon}\right)^{\vartheta}} .
\end{aligned}
$$

Thus, choosing $\vartheta \varepsilon>1$, we get

$$
\sum_{n=2}^{\infty} P\left(\left|Z_{n^{\varepsilon}}\right|>\sqrt{2 \vartheta \log \left(n^{\varepsilon}\right)}\right)<\infty .
$$

An application of the Borel-Cantelli lemma shows that

$$
\limsup _{n \rightarrow \infty} \frac{\left|Z_{n^{\varepsilon}}\right|}{\sqrt{2 \log \left(n^{\varepsilon}\right)}} \leq \sqrt{\vartheta} \quad \text { a.s. }
$$

Letting $\vartheta \rightarrow 1 / \varepsilon$ through the rational numbers, we get

$$
\limsup _{t \rightarrow \infty} \frac{Q\left(n^{\varepsilon}\right)}{\sqrt{2 \log n^{\varepsilon}}} \leqslant \frac{1}{\sqrt{\varepsilon}}\|f\|_{L^{2}} .
$$

Finally, (5.3), (5.4) and (5.5) allow us to conclude for the decomposition (5.2) that

$$
\limsup _{t \rightarrow \infty} \frac{|Q(t)|}{\sqrt{2 \log t}} \leq \frac{1}{\sqrt{\varepsilon}}\|f\|_{L^{2}} \quad \text { a.s. }
$$

Finally, by letting $\varepsilon \nearrow 1$ through the rational numbers, we get

$$
\limsup _{t \rightarrow \infty} \frac{|Q(t)|}{\sqrt{2 \log t}} \leq\|f\|_{L^{2}} \quad \text { a.s. }
$$

as required.

Proof of (a) in Theorem 3.1. By the variation of constants formula (2.6) the solution $Y$ of equation (2.5) can be decomposed according to

$$
\begin{aligned}
Y(t) & =y(t)+\sigma \int_{0}^{t} r(t-s) d B(s) \\
& =y(t)+\sigma Q(t)+\sigma c B(t),
\end{aligned}
$$

where $y$ is the solution of equation (2.1) and $Q$ is defined by

$$
Q(t)=\int_{0}^{t}(r(t-s)-c) d B(s) .
$$


We next notice that (2.2) and the fact that $r(t) \rightarrow c$ as $t \rightarrow \infty$ together imply that

$$
\lim _{t \rightarrow \infty} y(t)=\varphi(0) c+c \int_{[-\tau, 0]} \int_{s}^{0} \varphi(u) d u \nu(d s) .
$$

By combining the Law of the Iterated Logarithm for standard Brownian motion together Lemma 5.1 and (5.6), we find that

$$
\limsup _{t \rightarrow \infty} \frac{Y(t)}{\sqrt{2 t \log \log t}}=\sigma|c|, \quad \liminf _{t \rightarrow \infty} \frac{Y(t)}{\sqrt{2 t \log \log t}}=-\sigma|c| \quad \text { a.s. , }
$$

as required.

Lemma 5.2. Define for $\lambda>0$ and $k \in W^{2,1}\left(\mathbb{R}_{+}\right)$a Gaussian process $(K(t): t \geqslant 0)$ by

$$
K(t)=\int_{0}^{t} k(t-s) e^{-\lambda s} d B(s) .
$$

Then $\lim _{t \rightarrow \infty} K(t)=0$ a.s.

Proof. Applying the stochastic Fubini Theorem we obtain the representation

$$
\begin{aligned}
K(t) & =\int_{0}^{t}\left(k(0)+\int_{0}^{t-s} k^{\prime}(u) d u\right) e^{-\lambda s} d B(s) \\
& =k(0) \int_{0}^{t} e^{-\lambda s} d B(s)+\int_{0}^{t} \int_{0}^{v} k^{\prime}(v-s) e^{-\lambda s} d B(s) d v .
\end{aligned}
$$

Thus, for an arbitrary increasing sequence $\left(a_{n}\right)_{n=0}^{\infty}$ and $t \in\left[a_{n}, a_{n+1}\right)$ we have the identity

$$
K(t)=K\left(a_{n}\right)+k(0) \int_{a_{n}}^{t} e^{-\lambda s} d B(s)+\int_{a_{n}}^{t} \int_{0}^{v} k^{\prime}(v-s) e^{-\lambda s} d B(s) d v .
$$

Taking suprema over $\left[a_{n}, a_{n+1}\right]$ and using the Cauchy-Schwarz inequality lead to

$$
\begin{array}{r}
E\left[\sup _{a_{n} \leq t \leq a_{n+1}} K(t)^{2}\right] \leq 3 E\left[K\left(a_{n}\right)^{2}\right]+3 k(0)^{2} E\left[\sup _{a_{n} \leq t \leq a_{n+1}}\left(\int_{a_{n}}^{t} e^{-\lambda s} d B(s)\right)^{2}\right] \\
+3\left(a_{n+1}-a_{n}\right) \int_{a_{n}}^{a_{n+1}} E\left[\left(\int_{0}^{v} k^{\prime}(v-s) e^{-\lambda s} d B(s)\right)^{2}\right] d v .
\end{array}
$$

Since $k \in L^{2}\left(\mathbb{R}_{+}\right)$and $\lambda>0$ we have

$$
\int_{0}^{\infty} E\left[K^{2}(t)\right] d t=\int_{0}^{\infty} \int_{0}^{t} k^{2}(t-s) e^{-2 \lambda s} d s d t=\frac{1}{2 \lambda} \int_{0}^{\infty} k^{2}(u) d u<\infty .
$$

Lemma 3 in [4] enables us to choose a sequence $\left(a_{n}\right)_{n=0}^{\infty}$ with $a_{0}=0,0<a_{n+1}-a_{n}<1$ for all $n \in \mathbb{N}, \lim _{n \rightarrow \infty} a_{n}=\infty$ such that

$$
\sum_{n=0}^{\infty} E\left[K\left(a_{n}\right)^{2}\right]<\infty
$$


The sum over the second term in (5.7) can be estimated by applying Doob's inequality to obtain

$$
\sum_{n=0}^{\infty} E\left[\sup _{a_{n} \leq t \leq a_{n+1}}\left(\int_{a_{n}}^{t} e^{-\lambda s} d B(s)\right)^{2}\right] \leq \sum_{n=0}^{\infty} 4 \int_{a_{n}}^{a_{n+1}} e^{-2 \lambda s} d s<\infty .
$$

Applying Itô's isometry and letting $e_{2 \lambda}(t)=e^{-2 \lambda t}$ in the last term in (5.7), we arrive at

$$
\int_{a_{n}}^{a_{n+1}} E\left[\left(\int_{0}^{v} k^{\prime}(v-s) e^{-\lambda s} d B(s)\right)^{2}\right] d v=\int_{a_{n}}^{a_{n+1}}\left(k^{\prime 2} * e_{2 \lambda}\right)(v) d v .
$$

Since $k^{\prime 2}$ and $e_{2 \lambda}$ are in $L^{1}\left(\mathbb{R}_{+}\right)$, and by using the fact that $a_{n+1}-a_{n}<1$, it follows that

$$
\sum_{n=0}^{\infty}\left(a_{n+1}-a_{n}\right) \int_{a_{n}}^{a_{n+1}} E\left[\left(\int_{0}^{v} k^{\prime}(v-s) e^{-\lambda s} d B(s)\right)^{2}\right] d v \leqslant\left\|k^{\prime 2} * e_{2 \lambda}\right\|_{L^{1}}<\infty .
$$

Applying (5.8), (5.9) and (5.10) to the representation (5.7) gives

$$
\sum_{n=0}^{\infty} E\left[\sup _{a_{n} \leq t \leq a_{n+1}} K(t)^{2}\right]<\infty .
$$

Fubini's theorem implies

$$
\sum_{n=0}^{\infty} \sup _{a_{n} \leq t \leq a_{n+1}} K(t)^{2}<\infty \quad \text { a.s. }
$$

yielding $K(t) \rightarrow 0$ a.s. as $t \rightarrow \infty$.

Proof of (b) in Theorem 3.1. Define for all $t \geqslant 0$

$$
\begin{gathered}
k(t)=e^{-v_{0} t} r(t)-c, \\
K(t)=\int_{0}^{t} k(t-s) e^{-v_{0} s} d B(s) .
\end{gathered}
$$

By the variation of constants formula (2.6) we have

$$
e^{-v_{0} t} Y(t)=e^{-v_{0} t} y(t)+\sigma c \int_{0}^{t} e^{-v_{0} s} d B(s)+\sigma K(t) .
$$

The second term on the righthand side of (5.13) tends to the random variable $c \sigma \int_{0}^{\infty} e^{-v_{0} s} d B(s)$ as $t \rightarrow \infty$ a.s., by the martingale convergence theorem.

By (3.1) the function $k$ is in $L^{2}\left(\mathbb{R}_{+}\right)$. In order to prove that $k^{\prime}$ is also in $L^{2}\left(\mathbb{R}_{+}\right)$note that

$$
k^{\prime}(t)=-v_{0} e^{-v_{0} t} r(t)+\int_{[-\tau, 0]} e^{-v_{0}(t+s)} r(t+s) e^{v_{0} s} \nu(d s) .
$$

Because $v_{0}$ is a zero of $h$ we have

$$
k^{\prime}(t)=-v_{0}\left(e^{-v_{0} t} r(t)-c\right)+\int_{[-\tau, 0]}\left(e^{-v_{0}(t+s)} r(t+s)-c\right) e^{v_{0} s} \nu(d s) .
$$


Hence, by (3.1), we have that $k^{\prime} \in L^{2}\left(\mathbb{R}_{+}\right)$which enables us to apply Lemma 5.2 and to conclude $K(t) \rightarrow 0$ a.s. as $t \rightarrow \infty$.

For the first term in (5.13), the formula (2.2) yields

$$
\begin{aligned}
e^{-v_{0} t} y(t)= & \varphi(0) e^{-v_{0} t} r(t) \\
& +\int_{[-\tau, 0]} \int_{s}^{0} e^{-v_{0}(t+s-u)} r(t+s-u) e^{v_{0}(s-u)} \varphi(u) d u \nu(d s) \\
\rightarrow & \varphi(0) c+\int_{[-\tau, 0]} \int_{s}^{0} c e^{v_{0}(s-u)} \varphi(u) d u \nu(d s) \quad \text { as } t \rightarrow \infty .
\end{aligned}
$$

It remains to rearrange this limit, for which we consider

$$
\begin{aligned}
\int_{[-\tau, 0]} & \int_{s}^{0} e^{v_{0}(s-u)} \varphi(u) d u \nu(d s) \\
& =\int_{[-\tau, 0]} \int_{s}^{0} e^{v_{0}(s-u)}(\varphi(0)+\varphi(u)-\varphi(0)) d u \nu(d s) \\
& =-\frac{1}{v_{0}} \varphi(0) \int_{[-\tau, 0]}\left(e^{v_{0} s}-1\right) \nu(d s)+\int_{[-\tau, 0]} \int_{s}^{0} e^{v_{0}(s-u)}(\varphi(u)-\varphi(0)) d u \nu(d s) \\
& =-\frac{1}{v_{0}} \varphi(0)\left(v_{0}-0\right)+\int_{-\tau}^{0} e^{-v_{0} u}(\varphi(u)-\varphi(0))\left(\int_{[-\tau, u]} e^{v_{0} s} \nu(d s)\right) d u
\end{aligned}
$$

By applying this relation to (5.14) we obtain the desired formula.

\section{$6 \quad$ Proof of Theorem 3.3}

To prove Theorem 3.3, it is convenient to introduce the function

$$
F:[0, \tau] \rightarrow \mathbb{R}, \quad F(t)=\nu([-t, 0])
$$

and the function

$$
P: \mathbb{C} \rightarrow \mathbb{C}, \quad P(\lambda)=\int_{0}^{\tau} e^{-\lambda t} F(t) d t .
$$

Fubini's theorem and $\nu([-\tau, 0])=0$ yield

$$
P(\lambda)=\int_{[-\tau, 0]}\left(\int_{-s}^{\tau} e^{-\lambda t} d t\right) \nu(d s)=\frac{1}{\lambda} \int_{[-\tau, 0]} e^{\lambda s} \nu(d s)=-\frac{h(\lambda)}{\lambda}+1
$$

for $\lambda \neq 0$. Therefore, for $\lambda \neq 0$ we have that $P(\lambda)=1$ if and only if $h(\lambda)=0$. For $\lambda=0$ Fubini's theorem yields

$$
P(0)=\int_{[-\tau, 0]} \int_{-s}^{\tau} d t \nu(d s)=\int_{[-\tau, 0]} s \nu(d s)=m(\nu) .
$$

Proof of Theorem 3.3. (a): Because of (6.4), we have $P(0)>1$; and due to Lebesgue's dominated convergence theorem, we see $P(\lambda) \rightarrow 0$ as $\lambda \rightarrow \infty$. Differentiating $P$ and applying (3.5) results in

$$
P^{\prime}(\lambda)=-\int_{0}^{\tau} t e^{-\lambda t} F(t) d t<0 \quad \text { for all } \lambda \geq 0 .
$$


Hence, there exists a unique $\lambda_{0}>0$ such that $P\left(\lambda_{0}\right)=1$ and hence a unique $\lambda_{0}>0$ such that $h\left(\lambda_{0}\right)=0$. To see that this root is simple we differentiate $P$ by using representation (6.3) and obtain $P^{\prime}(\lambda)=-h^{\prime}(\lambda) / \lambda+h(\lambda) / \lambda^{2}$ for $\lambda>0$. Since $P^{\prime}\left(\lambda_{0}\right)<0$, and $P^{\prime}\left(\lambda_{0}\right)=$ $-h^{\prime}\left(\lambda_{0}\right) / \lambda_{0}$, we have $h^{\prime}\left(\lambda_{0}\right)>0$.

Suppose there exists $\lambda_{2} \in \mathbb{R}$ such that $h\left(\lambda_{0}+i \lambda_{2}\right)=0$. Then $P\left(\lambda_{0}+i \lambda_{2}\right)=1$ yields

$$
1=\int_{0}^{\tau} e^{-\lambda_{0} t} \cos \left(\lambda_{2} t\right) F(t) d t, \quad 0=\int_{0}^{\tau} e^{-\lambda_{0} t} \sin \left(\lambda_{2} t\right) F(t) d t .
$$

Since $h\left(\lambda_{0}\right)=0$, Fubini's Theorem implies that

$$
\frac{1}{\lambda_{0}} \int_{[-\tau, 0]} e^{\lambda_{0} s} \nu(d s)=\int_{0}^{\tau} e^{-\lambda_{0} t} F(t) d t=P\left(\lambda_{0}\right)=1 .
$$

Using this and the first equality in (6.5) give

$$
\int_{0}^{\tau} e^{-\lambda_{0} t}\left(1-\cos \left(\lambda_{2} t\right)\right) F(t) d t=0
$$

But because $F$ is non-negative and does not vanish Lebesgue almost everywhere, this yields $\lambda_{2}=0$.

Finally, we show that $\lambda_{1}<\lambda_{0}$ for all $\lambda_{1} \in\left(\mathbb{R}_{+}\right)$with $h\left(\lambda_{1}+i \lambda_{2}\right)=0$ for some $\lambda_{2} \in \mathbb{R}$. Because $P\left(\lambda_{1}+i \lambda_{2}\right)=1$ we have

$$
1=\operatorname{Re}\left(P\left(\lambda_{1}+i \lambda_{2}\right)\right)=\int_{0}^{\tau} e^{-\lambda_{1} t} \cos \left(\lambda_{2} t\right) F(t) d t \leq \int_{0}^{\tau} e^{-\lambda_{1} t} F(t) d t=P\left(\lambda_{1}\right) .
$$

Since $P$ is decreasing on $(0, \infty)$ and $P\left(\lambda_{0}\right)=1$, we must have $\lambda_{1} \leq \lambda_{0}$.

(b) The assumption $\nu([-\tau, 0])=0$ implies that $h$ has a root at 0 . The root is simple since $h^{\prime}(0)=1-\int_{[-\tau, 0]} s \nu(d s)>0$, using $m(\nu)<1$.

Suppose there exists $\lambda_{2} \neq 0$ such that $h\left(i \lambda_{2}\right)=0$. Then (6.3) implies $P\left(i \lambda_{2}\right)=1$ which results in

$$
1=\int_{0}^{\tau} \cos \left(\lambda_{2} t\right) F(t) d t, \quad 0=\int_{0}^{\tau} \sin \left(\lambda_{2} t\right) F(t) d t .
$$

On the other hand, (6.4) yields

$$
\int_{0}^{\tau} F(t) d t=P(0)=m(\nu)<1
$$

Consequently, by using the first equality in (6.7), we get

$$
\int_{0}^{\tau}\left(1-\cos \left(\lambda_{2} t\right)\right) F(t) d t<0
$$

which contradicts $F \geqslant 0$. Hence $h\left(i \lambda_{2}\right) \neq 0$ for all $\lambda_{2} \neq 0$.

By employing the same argument as in part (a), we may show that for all other roots $\lambda_{1}+i \lambda_{2}$ of $h$ we have $\lambda_{1}<0$.

\section{Proof of Theorem 3.5}

Lemma 7.1. Suppose that the signed measure $\nu$ satisfies

$$
\begin{aligned}
& \nu([-t, 0]) \geq 0 \quad \text { for all } t \in[0, \tau], \\
& \nu([-\tau, 0])=0
\end{aligned}
$$

and let $F$ be defined by $F(t)=\nu([-t, 0])$ for all $t \in[0, \tau]$. Then we have that: 
(a) $r^{\prime}(t) \geqslant 0$ for all $t \geqslant 0$;

(b) $m(\nu)<1$ implies $r^{\prime} \in L^{p}\left(\mathbb{R}_{+}\right)$for all $p \geqslant 1$;

(c) $m(\nu)>1$ implies $\lim _{t \rightarrow \infty} r^{\prime}(t) e^{-v_{0} t}=\left(\int_{0}^{\tau} s e^{-v_{0} s} F(s) d s\right)^{-1}$;

(d) $m(\nu)<1$ implies that there exists $\lambda_{0}>0$ such that

$$
\lim _{t \rightarrow \infty} r^{\prime}(t) e^{\lambda_{0} t}=\left(\int_{0}^{\tau} s e^{\lambda_{0} s} F(s) d s\right)^{-1} .
$$

Proof. (a) By considering first the case $t \in[0, \tau]$, we see that

$$
\begin{aligned}
r^{\prime}(t) & =\int_{[-t, 0]} r(t+s) \nu(d s) \\
& =\int_{[-t, 0]}\left(1+\int_{0}^{t+s} r^{\prime}(u) d u\right) \nu(d s) \\
& =F(t)+\int_{0}^{t} r^{\prime}(u) \int_{[u-t, 0]} \nu(d s) d u \\
& =F(t)+\int_{0}^{t} r^{\prime}(u) F(t-u) d u .
\end{aligned}
$$

On the other hand, if $t \geqslant \tau$, we have

$$
\begin{aligned}
r^{\prime}(t) & =\int_{[-\tau, 0]}\left(1+\int_{0}^{t+s} r^{\prime}(u) d u\right) \nu(d s) \\
& =\int_{0}^{t-\tau} r^{\prime}(u) \int_{[-\tau, 0]} \nu(d s) d u+\int_{t-\tau}^{t} r^{\prime}(u) \int_{[u-t, 0]} \nu(d s) d u \\
& =\int_{t-\tau}^{t} r^{\prime}(u) F(t-u) d u .
\end{aligned}
$$

By letting $F(t)=0$ for $t \geqslant \tau$, we can summarise (7.1) and (7.2) as

$$
r^{\prime}(t)=F(t)+\int_{0}^{t} r^{\prime}(t-u) F(u) d u \quad \text { for all } t \geqslant 0 .
$$

Because $F(t) \geqslant 0$ for all $t \geqslant 0$, the renewal theorem implies that $r^{\prime}(t) \geqslant 0$ for all $t \geqslant 0$. (b) By applying Fubini's Theorem, we obtain the identity

$$
\int_{0}^{\infty} F(t) d t=\int_{0}^{\tau} \int_{[-t, 0]} \nu(d s) d t=\int_{[-\tau, 0]} \int_{-s}^{\tau} d t \nu(d s)=\int_{[-\tau, 0]} s \nu(d s)=m(\nu) .
$$

Since $r^{\prime}$ satisfies the renewal equation (7.3), we have

$$
\left(1-\|F\|_{L^{1}}\right) \int_{0}^{\infty} r^{\prime}(u) d u \leqslant\|F\|_{L^{1}},
$$

which yields $r \in L^{1}\left(\mathbb{R}_{+}\right)$because $\|F\|_{L^{1}}<1$. Once we have proved (d), we know that $r^{\prime}(t) \rightarrow 0$ for $t \rightarrow \infty$, demonstrating that $r^{\prime}$ is bounded. Consequently, $r^{\prime}$ is in $L^{p}\left(\mathbb{R}_{+}\right)$ for every $p \geqslant 1$. 
(c) Since $v_{0}>0$ is a root of $h$ we obtain

$$
\int_{0}^{\tau} F(s) e^{-v_{0} s} d s=\int_{[-\tau, 0]} \int_{-u}^{\tau} e^{-v_{0} s} d s \nu(d u)=\frac{1}{v_{0}} \int_{[-\tau, 0]} e^{v_{0} u} \nu(d u)=1 .
$$

Consequently, $\rho(d s):=F(s) \exp \left(-v_{0} s\right) d s$ defines a probability measure and (7.3) implies that the function $s \mapsto g(s):=r^{\prime}(s) \exp \left(-v_{0} s\right)$ is a solution of

$$
g(t)=e^{-v_{0} t} F(t)+\int_{0}^{t} g(t-s) \rho(d s) \quad \text { for all } t \geqslant 0 .
$$

The renewal theorem establishes the limit in (c).

(d) If we define the function

$$
G:[0, \infty) \rightarrow \mathbb{R}, \quad G(\lambda):=\int_{0}^{\tau} F(s) e^{\lambda s} d s
$$

then it follows by (7.4) that $G(0)=m(\nu)<1$. On the other hand, Lebesgue's dominated convergence theorem shows that $G(\lambda) \rightarrow \infty$ for $\lambda \rightarrow \infty$. Thus, there exists $\lambda_{0}>0$ such that $G\left(\lambda_{0}\right)=1$, which means that $\rho(d s):=F(s) \exp \left(\lambda_{0} s\right) d s$ defines a probability measure. Now applying the renewal theorem completes the proof as in part (c).

Proof of Theorem 3.5. (a) By the representation (3.8) for $t \geq \delta$ we have

$$
\begin{aligned}
\operatorname{Cov}\left(Y_{\delta}(t), Y_{\delta}(t+\Delta)\right) & =E\left[\int_{0}^{t} r_{\delta}(t-s) \sigma d B(s) \cdot \int_{0}^{t+\Delta} r_{\delta}(t+\Delta-s) \sigma d B(s)\right] \\
& =\sigma^{2} \int_{0}^{t} r_{\delta}(t-s) r_{\delta}(t+\Delta-s) d s \\
& =\sigma^{2} \int_{0}^{t} r_{\delta}(u) r_{\delta}(u+\Delta) d u
\end{aligned}
$$

Applying part (a) of Lemma 7.1 completes the proof of (a).

(b) Combining $r^{\prime}(t) \rightarrow 0$ as $t \rightarrow \infty$, which is due to part (d) of Lemma 7.5, and

$$
r_{\delta}(t)=r(t)-r(t-\delta)=\int_{t-\delta}^{t} r^{\prime}(s) d s \quad \text { for } t \geq \delta,
$$

implies $r_{\delta}(t) \rightarrow 0$ as $t \rightarrow \infty$. Also for $T \geq 2 \delta$ we have

$$
\begin{aligned}
\int_{\delta}^{T} r_{\delta}(t) d t & =\int_{\delta}^{T} \int_{t-\delta}^{t} r^{\prime}(s) d s d t \\
& =\int_{0}^{\delta} \int_{\delta}^{s+\delta} d t r^{\prime}(s) d s+\int_{\delta}^{T-\delta} \int_{s}^{s+\delta} d t r^{\prime}(s) d s+\int_{T-\delta}^{T} \int_{s}^{T} d t r^{\prime}(s) d s \\
& =\int_{0}^{\delta} s r^{\prime}(s) d s+\int_{\delta}^{T-\delta} \delta r^{\prime}(s) d s+\int_{T-\delta}^{T}(T-s) r^{\prime}(s) d s \\
& \leq \delta \int_{0}^{T} r^{\prime}(s) d s .
\end{aligned}
$$

Therefore, $r_{\delta} \in L^{1}\left(\mathbb{R}_{+}\right)$for each $\delta>0$. The representation (7.5) yields the limit

$$
c_{\delta}(\Delta):=\lim _{t \rightarrow \infty} \operatorname{Cov}\left(Y_{\delta}(t), Y_{\delta}(t+\Delta)\right)=\sigma^{2} \int_{0}^{\infty} r_{\delta}(u) r_{\delta}(u+\Delta) d u
$$


and $r_{\delta} \in L^{1}\left(\mathbb{R}_{+}\right)$together with the boundedness of $r_{\delta}$ guarantees that the limit is finite. The estimate

$$
0 \leq c_{\delta}(\Delta) \leq \sigma^{2}\left\|r_{\delta}\right\|_{L^{1}} \sup _{v \geq \Delta} r_{\delta}(v)
$$

shows that $c_{\delta}(\Delta) \rightarrow 0$ as $\Delta \rightarrow \infty$. The convolution integral in (7.6) yields that

$$
\int_{0}^{\infty} c_{\delta}(\Delta) d \Delta=\sigma^{2} \int_{0}^{\infty} r_{\delta}(u)\left(\int_{0}^{\infty} r_{\delta}(v) d v\right) d u \leq \sigma^{2}\left\|r_{\delta}\right\|_{L^{1}}^{2}
$$

and so $c_{\delta} \in L^{1}\left(\mathbb{R}_{+}\right)$.

(c) Part (d) of Lemma 7.1 implies that there exists a $\lambda_{0}>0$ such that

$$
\lim _{t \rightarrow \infty} r^{\prime}(t) e^{\lambda_{0} t}=\left(\int_{0}^{\tau} t e^{\lambda_{0} t} F(t) d t\right)^{-1}=: R .
$$

Since we have

$$
\begin{aligned}
r_{\delta}(t) e^{\lambda_{0} t} & =\int_{t-\delta}^{t} r^{\prime}(s) e^{\lambda_{0} s} \cdot e^{\lambda_{0}(t-s)} d s \\
& =\int_{t-\delta}^{t}\left(r^{\prime}(s) e^{\lambda_{0} s}-R\right) \cdot e^{\lambda_{0}(t-s)} d s+R \int_{t-\delta}^{t} e^{\lambda_{0}(t-s)} d s \\
& =\int_{0}^{\delta}\left(r^{\prime}(s+t-\delta) e^{\lambda_{0}(s+t-\delta)}-R\right) e^{\lambda_{0}(\delta-s)} d s+R \int_{0}^{\delta} e^{\lambda_{0} s} d s,
\end{aligned}
$$

it follows by Lebesgue's dominated convergence theorem that

$$
\lim _{t \rightarrow \infty} r_{\delta}(t) e^{\lambda_{0} t}=R \int_{0}^{\delta} e^{\lambda_{0} s} d s .
$$

The representation (7.6) yields

$$
\begin{aligned}
c_{\delta}(\Delta) e^{\lambda_{0} \Delta} & =\sigma^{2} \int_{0}^{\infty} r_{\delta}(u) e^{-\lambda_{0} u} r_{\delta}(u+\Delta) e^{\lambda_{0}(\Delta+u)} d u \\
& \rightarrow \sigma^{2} R\left(\int_{0}^{\delta} e^{\lambda_{0} u} d u\right)\left(\int_{0}^{\infty} r_{\delta}(u) e^{-\lambda_{0} u} d u\right) \quad \text { as } \Delta \rightarrow \infty,
\end{aligned}
$$

which finishes the proof.

\section{Proof of Theorem 4.1}

In (5.14) we derived that

$$
\lim _{t \rightarrow \infty} e^{-v_{0} t} y(t)=E[\Gamma(\varphi)],
$$

where $y=y(\cdot, \varphi)$ is the solution of $(2.1)$. Thus, to show the claim in (a), we have to determine the sign of the limit.

We extend the measure $\mu$ by setting $\mu(I):=0$ for all Borel sets $I \subseteq(-\infty,-\tau)$. According to property (b) in Section 4.2.2, the distribution of $\Gamma(\varphi)$ is invariant under affine 
transformation of $\varphi$; thus, we can assume $\varphi(0)=0$ in the sequel. Using integration by parts we can rewrite equation (2.1) for all $t \in[0, \tau]$ according to

$$
\begin{aligned}
y^{\prime}(t) & =\int_{(-t, 0]} y(t+s) \nu(d s)+\int_{[-\tau,-t]} y(t+s) \nu(d s) \\
& =\nu((-\infty, 0]) y(t)-\nu((-\infty,-t]) \varphi(0)-\int_{-t}^{0} y^{\prime}(t+s) \nu((-\infty, s]) d s+\int_{[-\tau,-t]} y(t+s) \nu(d s) \\
& =\int_{-t}^{0} y^{\prime}(t+s) \nu((s, 0]) d s+\int_{[-\tau,-t]} y(t+s) \nu(d s),
\end{aligned}
$$

where we used in the last line the assumptions $\nu([-\tau, 0])=0$ and $\nu([-\tau, s])+\nu((s, 0])=0$ for all $s \in[-\tau, 0]$. For every $t>\tau$, one obtains analogously that

$$
y^{\prime}(t)=\int_{[-\tau, 0]} y(t+s) \nu(d s)=\int_{-\tau}^{0} y^{\prime}(t+s) \nu((s, 0]) d s .
$$

By defining the function

$$
g(t):= \begin{cases}0, & \text { if } t>\tau, \\ \int_{[-\tau,-t]} \varphi(t+s) \nu(d s), & \text { if } t \in[0, \tau],\end{cases}
$$

the two cases can be consolidated to give

$$
y^{\prime}(t)=\int_{-t}^{0} y^{\prime}(t+s) \nu((s, 0]) d s+g(t) \quad \text { for all } t \geqslant 0 .
$$

Since $m(\nu)>1$ we have that $\int_{-\tau}^{0} e^{v_{0} s} \nu((s, 0]) d s=1$ and thus,

$$
y^{\prime}(t) e^{-v_{0} t}=\int_{-t}^{0} y^{\prime}(t+s) e^{-v_{0}(t+s)} e^{v_{0} s} \nu((s, 0]) d s+e^{-v_{0} t} g(t), \quad t \geqslant 0,
$$

is a proper renewal equation. The renewal theorem implies that

$$
\lim _{t \rightarrow \infty} \frac{y^{\prime}(t)}{e^{v_{0} t}}=\frac{\int_{0}^{\tau} e^{-v_{0} s} g(s) d s}{\int_{-\tau}^{0} s e^{v_{0} s} \nu((s, 0]) d s},
$$

which, by applying l'Hôpital's rule, results in

$$
\lim _{t \rightarrow \infty} \frac{y(t)}{e^{v_{0} t}}=\frac{\int_{0}^{\tau} e^{-v_{0} s} g(s) d s}{\lambda \int_{-\tau}^{0} s e^{v_{0} s} \nu((s, 0]) d s} .
$$

Since the denominator is positive by condition (3.5), we focus on the numerator on the right hand side of (8.1). We begin by showing that $g$ is non-negative. Applying integration by parts results in

$$
g(t)=-\int_{-\tau}^{-t} \varphi^{\prime}(t+s) \nu((-\infty, s]) d s=\int_{-\tau}^{-t} \varphi^{\prime}(t+s) \nu((s, 0]) d s
$$

for each $t \in[0, \tau]$ which shows $g(t) \geqslant 0$ for all $t \geqslant 0$. Moreover, since $\varphi^{\prime}(0)>0$ there exist two constants $c, \delta>0$ such that

$$
g(t) \geqslant \int_{-t-\delta}^{-t} \varphi^{\prime}(t+s) \nu((s, 0]) d s \geqslant c \int_{-t-\delta}^{-t} \nu((s, 0]) d s \quad \text { for all } t \geqslant 0 .
$$


Since $s \mapsto \nu((s, 0])$ is right-continuous and $\nu \neq 0$ it follows that there exists $t_{0} \geqslant 0$ such that $g\left(t_{0}\right)>0$. The right-continuity of $g$ yields that the numerator in (8.1) is strictly positive which completes the proof.

Part (b) can be proved analogously.

\section{References}

[1] V. Anh and A. Inoue, Financial markets with memory. I. Dynamic models, Stoch. Anal. Appl., 23 (2), 275-300, 2005.

[2] V. Anh, A. Inoue and Y. Kasahara, Financial markets with memory. II. Innovation processes and expected utility maximization, Stoch. Anal. Appl., 23 (2), 301-328, 2005.

[3] M. Arriojas, Y. Hu, S.-E. Mohammed and G. Pap, A delayed Black and Scholes formula, Stoch. Anal. Appl., 25 (2), 471-492, 2007.

[4] J. A. D. Appleby, $p^{t h}$-mean integrability and almost sure asymptotic stability of Itô-Volterra equations, J. Integral Equations Appl., 15 (4), 321-341, 2003.

[5] J. A. D. Appleby, X. Mao and H. Wu, On the almost sure running maxima of solutions of affine stochastic functional differential equations, SIAM J. Math. Anal., $42(2), 646-678,2010$.

[6] J.-P. Bouchaud and R. Cont, A Langevin approach to stock market fluctuations and crashes, Eur. Phys. J. B, 6, 543-550, 1998.

[7] P. Cheridito, Gaussian moving averages, semimartingales and option pricing, Stochastic Processes Appl., 109 (1), 47-68, 2004

[8] P. H. Cootner, The random character of stock market prices, Cambridge: MIT Press, 1964.

[9] J. B. De Long, A. Shleifer, L. H. Summers and R. J. Waldmann, Noise trader risk in financial markets, J. Political Economy, 98, 703-738, 1990.

[10] O. Diekmann, S.A. van Gils, S.M. Verduyn Lunel and H.-O. Walther, Delay equations. Functional-, Complex-, and Nonlinear Analysis, New York: Springer, 1995.

[11] E. F. Fama, Efficient capital markets: a review of theory and empirical work, Journal of Finance, 25 (2), 383-417, 1970.

[12] H. Föllmer and M. Schweizer, A microeconomic approach to diffusion models for stock prices, Mathematical Finance, 3, 1-23, 1993.

[13] A. A. Gushchin and U. Küchler, On stationary solutions of delay differential equations driven by a Lévy process, Stochastic Processes Appl., 88 (2), 195-211, 2000.

[14] G. Gripenberg, S.-O. Londen, and O. Staffans, Volterra integral and functional equations, Cambridge: Cambridge University Press, 1990.

[15] D. Hobson and L. C. G. Rogers, Complete models with stochastic volatility, Mathematical Finance, 8, 27-48, 1998. 
[16] D. Kahneman and M. R. Riepe, Aspects of investor psychology, J. Portfolio Management, 24, 52-65, 1998.

[17] A. Lo and A. C. MacKinlay, A Non-random walk down Wall Street, Princeton: Princeton University Press, 1999.

[18] X. Mao, Stochastic differential equations and their applications, Chichester: Horwood Publishing, 1997.

[19] S. N. Neftci, Naive trading rules in financial markets and Wiener-Kolmogorov prediction: a study of technical analysis, J. Business, 64 (4), 549-571, 1991.

[20] A. Orléan, Mimetic contagion and speculative bubbles, Theory and Decisions, 27, 63-92, 1989 .

[21] P. E. Protter, Stochastic integration and differential equations. New York: Springer, 2004.

[22] M. Reiß, M. Riedle and O. van Gaans, On Emery's inequality and a variation-ofconstants formula, Stochastic Anal. Appl., 25 (2), 353 - 379, 2007. 